\title{
Resolve cathode electrolyte interphase in lithium batteries with cryo-EM
}

Zewen Zhang ${ }^{1}$, Jinlong Yang ${ }^{2}$, William Huang ${ }^{1}$, Hansen Wang ${ }^{3}$, Weijiang Zhou ${ }^{4}$, Yanbin Li ${ }^{3}$, Yuzhang $\mathrm{Li}^{5}$, Jinwei $\mathrm{Xu}^{3}$, Wenxiao Huang ${ }^{3}$, Wah $\mathrm{Chiu}^{4}$ and $\mathrm{Yi} \mathrm{Cui}^{3}$

${ }^{1}$ Department of Materials Science and Engineering, Stanford University, stanford, California, United States, ${ }^{2}$ School of Materials Science and Engineering, Shenzhen University, Shenzhen, Guangdong, China (People's Republic), ${ }^{3}$ Department of Materials Science and Engineering, Stanford University, Stanford, California, United States, ${ }^{4}$ School of Medicine, Stanford University, Stanford, California, United States, ${ }^{5}$ Department of Chemical and Biomolecular Engineering, University of California, Los Angeles, Los Angeles, California, United States

Lithium (Li)-based battery technology has revolutionized modern energy storage and is critical for a decarbonized future [1]. However, batteries are complex and further development entails a nanoscopic understanding of the structure, chemistry and function of the interfaces in the battery. Most well-known among these the important yet sensitive battery interfaces is the solid-electrolyte interphase (SEI), an interfacial layer forms on the electrodes[2]. This SEI has been identified as the key component that determines the cycle stability and the failure mode of the battery [3]. Unfortunately, characterization tools with high spatial resolution, such as transmission electron microscopy (TEM), are not compatible with SEI since these interphases are highly sensitive to air, moisture, and electron beam radiation. Moreover, SEI on the positive electrode (cathode), or cathode electrolyte interphase (CEI), has been even less wellstudied compared to the negative electrode (anode) SEI [4]. Limited information about CEI is thus derived from surface-analytical techniques with poor in-plane resolution such as X-ray photoelectron spectroscopy (XPS) and Raman spectroscopy, and the existence of CEI is often described as a passivation coating layer on cathode particles, as an exact analogue to its anode counterpart.

Recent development in cryogenic TEM (cryo-TEM) for physical science has made possible the atomic resolution imaging as well as the nanoscale elemental mapping of sensitive battery materials in TEM without side reactions [5,6,7]. Here, using cryo-TEM and cryogenic scanning TEM (cryo-STEM), we resolve the cathode electrolyte interface in their native state. We find that under normal operation conditions, there does not exist an intimate coating layer at the single-particle level in commercial carbonate-based electrolyte on cathodes [Fig. 1]. This generates a different perspective about CEI than previously understood. However, we also found that upon brief external electrical shorting between anodes and cathodes, a solid-electrolyte interphase (SEI), which usually forms at the anode surface, could form on cathodes, and be electrochemically converted into a stable, conformal CEI in situ [Fig. 2]. With electron energy loss spectroscopy (EELS), we identify the composition and map out the distribution of CEI species in this conformal layer. High spatial resolution techniques such as cryo-(S)TEM help resolve the nanoscopic picture of CEI, which cannot be accessed through surface analytical methods. This work reveals the heterogeneity in battery interfaces between nanoscale and microscale, as well as highlights the importance of atomic scale characterization of battery interphases in native state. 

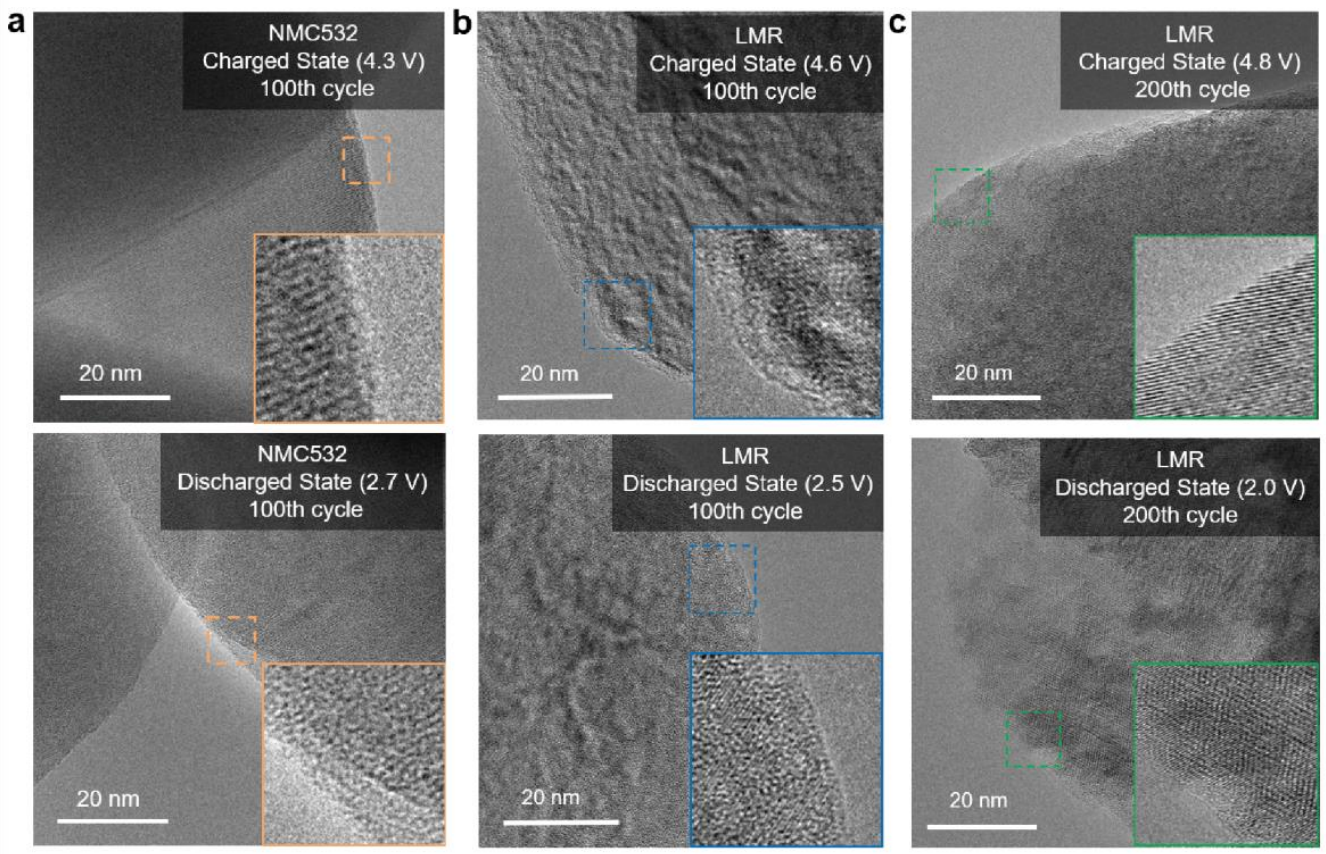

Figure 1. Figure 1. Cryo-HRTEM images of cathode electrolyte interface under normal cycle conditions on NMC532 and Li, Mn-rich NMC532 (LMR) positive electrodes in commercial carbonate-based electrolyte (1 M LiPF6 in EC/DEC). (a) Cryo-HRTEM images of the interface on NMC532 positive electrode cycled between $2.7-4.3 \mathrm{~V}$ after 100 cycles at a rate of $0.5 \mathrm{C}$ in both charged and discharged state. (b) Cryo-HRTEM images of the interface on LMR positive electrode cycled between $2.5-4.6 \mathrm{~V}$ after 100 cycles at a rate of $0.5 \mathrm{C}$ in both charged and discharged state. (c) Cryo-HRTEM images of the interface on LMR positive electrode cycled between $2.0-4.8 \mathrm{~V}$ after 200 cycles at a rate of $0.5 \mathrm{C}$ in both charged and discharged state. There is no intimate coating layer on the interface in all conditions. All images are $70 \mathrm{~nm}$ wide.
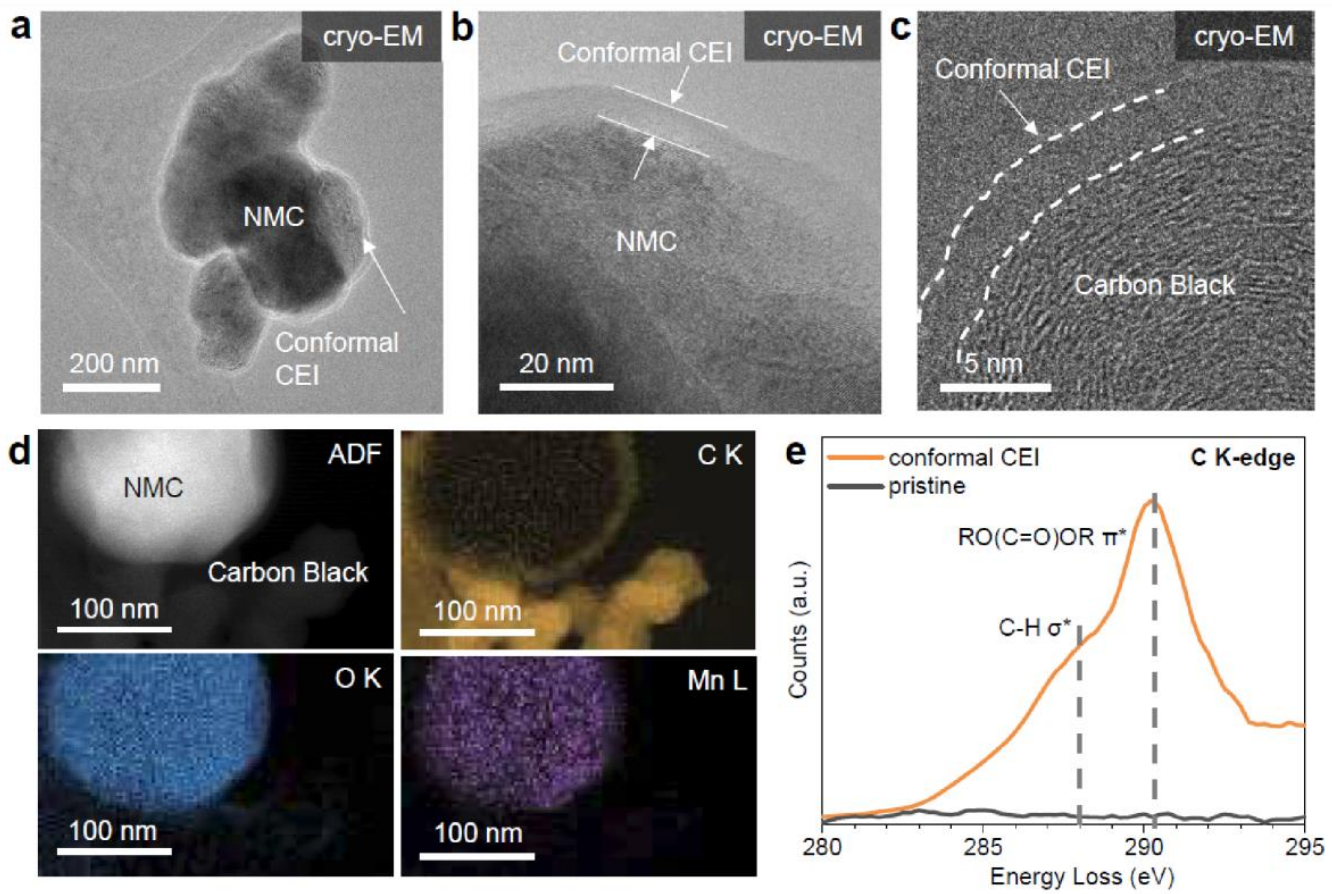
Figure 2. Figure 2. Cryo-(S)TEM images of conformal CEI formed on NMC532 positive electrodes in commercial carbonate-based electrolyte (1 M LiPF6 in EC/DEC). (a) Cryo-TEM image of the conformal CEI on NMC532 positive electrode. Uniform intimate coating layer could be observed. The image is 800 $\mathrm{nm}$ wide. (b) cryo-HRTEM image of the conformal CEI directly interfaced with NMC532 positive electrode particle. The layer is about $10 \mathrm{~nm}$ thick and mainly amorphous. The image is $80 \mathrm{~nm}$ wide. (c) cryo-HRTEM image of the conformal CEI on carbon black additives. The image is $20 \mathrm{~nm}$ wide. (d) cryo-STEM ADF image and EELS mapping of C, O, Mn of a representative region. Window is $300 \mathrm{~nm}$ x $180 \mathrm{~nm}$. (e) EELS fine structure of carbon K-edge at the interface in pristine positive electrode and conformal CEI.

References

[1] D. Larcher and J.-M. Tarascon, "Towards greener and more sustainable batteries for electrical energy storage," Nat. Chem., vol. 7, no. 1, Art. no. 1, Jan. 2015, doi: 10.1038/nchem.2085.

[2] E. Peled, "The Electrochemical Behavior of Alkali and Alkaline Earth Metals in Nonaqueous Battery Systems-The Solid Electrolyte Interphase Model," J. Electrochem. Soc., vol. 126, no. 12, pp. 2047-2051, Dec. 1979, doi: 10.1149/1.2128859.

[3] E. Peled and S. Menkin, "Review_SEI: Past, Present and Future," J. Electrochem. Soc., vol. 164, no. 7, pp. A1703-A1719, Jan. 2017, doi: 10.1149/2.1441707jes.

[4] K. Xu, "Electrolytes and Interphases in Li-Ion Batteries and Beyond," Chem. Rev., vol. 114, no. 23, pp. 11503-11618, Dec. 2014, doi: 10.1021/cr500003w.

[5] M. J. Zachman, Z. Tu, S. Choudhury, L. A. Archer, and L. F. Kourkoutis, "Cryo-STEM mapping of solid-liquid interfaces and dendrites in lithium-metal batteries," Nature, vol. 560, no. 7718, pp. 345349, Aug. 2018, doi: 10.1038/s41586-018-0397-3.

[6] Y. Li et al., "Atomic structure of sensitive battery materials and interfaces revealed by cryoelectron microscopy," Science, vol. 358, no. 6362, pp. 506-510, Oct. 2017, doi: 10.1126/science.aam6014.

[7] X. Wang et al., "New Insights on the Structure of Electrochemically Deposited Lithium Metal and Its Solid Electrolyte Interphases via Cryogenic TEM," Nano Lett., vol. 17, no. 12, pp. 7606-7612, Dec. 2017, doi: 10.1021/acs.nanolett.7b03606. 